\title{
Review on Intermolecular Forces between Dyes Used for Polyester Dyeing and Polyester Fiber
}

\author{
Asnake Ketema $(\mathbb{D}$ and Amare Worku \\ Department of Textile Engineering, Dire Dawa Institute of Technology, Dire Dawa University, Dire Dawa, Ethiopia \\ Correspondence should be addressed to Amare Worku; amareworku2005@ddu.edu.et
}

Received 8 October 2020; Revised 24 October 2020; Accepted 28 October 2020; Published 11 November 2020

Academic Editor: Marinos Pitsikalis

Copyright (C 2020 Asnake Ketema and Amare Worku. This is an open access article distributed under the Creative Commons Attribution License, which permits unrestricted use, distribution, and reproduction in any medium, provided the original work is properly cited.

Polyester fiber is a manufactured fiber composed of synthetic linear macromolecules in the chain at least $85 \%$ by mass of an ester of diol and benzene-1, 4-dicarboxylic acid (terephthalic acid). Fibers of the most common polyester, poly(ethylene terephthalate) (PET or PET), are generally made from either terephthalic acid or dimethyl terephthalate together with ethylene glycol. Dyeing of polyester fabric with disperse dyes, polyester requires the use of dispersing agents. The chemical characteristics and general application conditions of disperse dyes are characterized by the absence of solubilizing groups and low molecular weight. Dye particles have a size ranging from 0.5 to 2.0 microns, generally contain $-\mathrm{NH} 2$, substituted $-\mathrm{NH}_{2}$, or $-\mathrm{OH}$ groups in the structure, and get attached with the fiber through $\mathrm{H}$-bond and van der Waals force. Dyes are retained by the fiber by physical forces. Fastness properties are very high in polyester except in certain cases. The dye-fiber (PET) affinity is the result of different types of interactions, such as hydrogen bonds, dipole-dipole interactions, and van der Waals forces.

\section{Introduction}

Polyester fiber is a manufactured fiber composed of synthetic linear macromolecules in the chain at least $85 \%$ by mass of an ester of diol and benzene-1, 4-dicarboxylic acid (terephthalic acid). Fibers of the most common polyester, poly(ethylene terephthalate) (PET or PET), made from either terephthalic acid or dimethyl terephthalate together with ethylene glycol (Figure 1) [1].

The polyester term is usually used for polyethylene terephthalate (PET), despite the fact that the numerous polyester forms are present. It contains methylene (-CH2-), carbonyl $(=\mathrm{CO})$, and ester group (COOR) in their structure $[2,3]$. Polyester fiber has a highly compacted and crystalline structure which can resist dye uptake and more hydrophobic. For this reason, aqueous dyeing is carried out at high temperature and pressure by using dispersed dyes. Dyeing polyesters with disperse dyes require the use of dispersing agents. While beginners happily charge for dyeing with acid dyes for wool or nylon, and fiber, reactive dyes such as cotton and rayon, often with excellent results, the immersion dyeing of polyester is a different story. However, dispersed dye can be used by even young children to make designed son paper, which can be transferred to polyester fabrics or other synthetics with hot iron. The possibilities are endless using fabric crayons, rubber stamps, painting, and even screen printing [4]. The chemical characteristics and general application conditions of disperse dyes are characterized by the absence of solubilizing groups and low molecular weight. From a chemical point of view, more than $50 \%$ of the dispersed dyes are simple azo compounds, about $25 \%$ are anthraquinones, and the rest are methane, nitro, and naphtha-quinone dyes [4].

Dyeing polyester fiber using microwave irradiation was investigated $[5,6]$. Microwave irradiation to heat the dye bath. Then, Increased levels of dye uptake compared to conventional polyester dyeing method. UV irradiation Treatment of polyester fabric gives good color strength and fastness properties when irradiated polyester ( 90 minutes) at $100^{\circ} \mathrm{C}$ for 60 minutes using dyeing media of $\mathrm{pH} 10$ in the presence of $6 \mathrm{~g} / \mathrm{L}$ of dispersing agent using unirradiated disperse dyes $[7,8]$. 


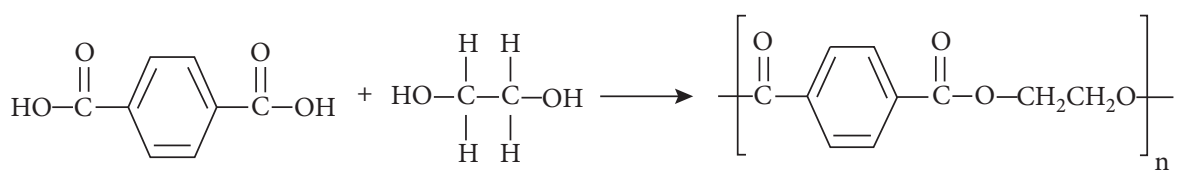

FIgURE 1: The polymerization reaction of terephthalic acid and ethylene glycol.

\section{Dyeing of Polyesters}

Dyeing synthetic fibers is a large subject in its own right. The readers are advised to consult one of the many publications that deal with it comprehensively. When PET fibers first appeared, they presented many problems for traditional dyers. PET has no functional groups to give affinity for usual dyestuffs. Natural fibers like wool, cotton, silk, and then later manmade ones like rayon and nylon were well known and had good dye affinity because the fibers had pendant or terminal functional chemical groups such as $-\mathrm{NH}_{2}$, $-\mathrm{COOH}$, and $-\mathrm{OH}$. These dyes were developed to interact with such groups. The only way to dye polyester was to rely on van der Waals forces to hold the dye in the fiber. This dye has little or no affinity for PET [9].

\subsection{The Dye-Fiber (PET) Affinity Is the Result of Different Types of Interactions [10]}

\section{Hydrogen bonds \\ Dipole-dipole interaction \\ van der Waals forces}

Disperse dyes have hydrogen atoms in their molecules, which are capable of forming hydrogen bonds with oxygen and nitrogen atoms in the fiber.

Dipole-dipole interactions result from the asymmetrical structure of the dye molecules, which makes possible electrostatic interactions between dipoles on the dye molecules and polarized bonds on the fiber. It is widely held that the substantive use of dispersed dyes towards PET fibers can be attributed to a variety of intermolecular forces and that $\mathrm{H}$-bonding predominates, although van der Waals forces also contribute $[11,12]$.

Hydrogen bonds (H-bonds) are highly complex phenomena that have been the subject of a great deal of research over many years. Three types of $\mathrm{H}$-bonds are available, namely, weak $\left(160 \mathrm{~kJ} \cdot \mathrm{mol}^{-1}\right.$; moderate (16 to $\left.60 \mathrm{~kJ} \cdot \mathrm{mol}^{-1}\right)$, and strong $\left(60\right.$ to $>160 \mathrm{~kJ} \cdot \mathrm{mol}^{-1}$. The three classes of $\mathrm{H}$-bonds also generally vary in terms of bond length (weak $>$ moderate $>$ strong) and bond angle (weak $<$ moderate $<$ strong). The attractive, predominantly electrostatic $\mathrm{H}$-bonding interaction is commonly expressed in the form $\mathrm{A}-\mathrm{H}: \mathrm{B}$, where $\mathrm{A}-\mathrm{H}$ is a polar donor bond (i.e., $\mathrm{A}_{\delta^{-}}-\mathrm{H}_{\delta+}$ ) and $\mathrm{B}$ is an acceptor atom $\mathrm{B}_{\delta^{-}}$ with both $\mathrm{A}$ and $\mathrm{B}$ are strongly electronegative elements and $\mathrm{B}$ has a lone pair of electrons. A feature of $\mathrm{H}$-bonds is their ability to cooperate, utilizing which H-bonds can reinforce each other resulting in the formation of regular $\mathrm{H}$-bonded structures, as exemplified by protein fibers (e.g., wool), PA 6 and PA 66 fibers, in which H-bonds are formed between - CONH groups and polyester fibers of adjacent polymer chains.

2.1.1. van der Waals Interactions (Also Known as van der Waals Forces). To account for some properties of nonideal gases and liquids, van der Waals introduced the concept of a general attractive interaction between neutral atoms in 1873 . As three related, but different, phenomena were subsequently found to contribute to these interactions, the term van der Waals interactions (aka van der Waals forces) is a collective name that describes a group of interactions [9]. The fundamental elements of these interactions are the dipole moment (uneven charge distribution within a molecule) and Polaris ability (the relative tendency of charge redistribution within a molecule imparted by an electric field). van der Waals' forces differ from other kinds of intermolecular forces in that they are always involved in interactions between bodies, whereas other types of interaction depend upon a particular feature such as electric charge (e.g., ion-ion forces). van der Waals forces may be either attractive forces, arising from interactions between the partial electric charges of polar molecules, or repulsive forces, which prevent the collapse of all molecules. They result from the exclusion of electrons from areas of electron orbital overlap. As mentioned, there are three types of forces that contribute to this long-range interaction between polar molecules, which are collectively known as van der Waals interactions [13]:

(1) Dipole-dipole (orientation) interactions (as formulated by Keesom)

(2) Dipole-induced dipole (induction) interactions (as formulated by Debye)

(3) Induced dipole-induced dipole (dispersion) interactions (as formulated by London)

Of these, while both the Keesom and Debye interactions occur in the case of molecules that possess permanent dipole moments, the London interaction is universal and occurs between atoms.

The energy of interaction of each of the three forces varies with dispersion interactions dominating except for very small molecules such as water. The use of the term van der Waals is often (and confusingly) used to describe only induced dipole-induced dipole interactions, whereas, as mentioned, the total van der Waals interaction constitutes the three contributions listed above.

Natural fibers such as wool, cotton, and silk (and later nylon, synthetic) were well understood and they had good dye attraction, owing to multiple fiber functionalities such as $-\mathrm{NH}_{2},-\mathrm{COOH}$, and $-\mathrm{OH}$. The only way to dye polyester are 
to first force the dye into the fiber and then rely on van der Waals forces to hold the dye in place. Classical cationic and anionic dyes for wool and silk or direct dyes for cotton all had water-solubilizing groups such as $-\mathrm{NR}_{3}+$ and $-\mathrm{SO}_{3}$ groups. Such dyes have little or no affinity for the hydrophobic PET [14].

\subsubsection{Hydrophobic Effect and Hydrophobic Interactions.} The hydrophobic effect concerns the very low miscibility of nonpolar substances in water, while the hydrophobic interaction describes the unusually strong attraction between hydrophobic molecules and surfaces in water that results in nonpolar molecules (e.g., alkenes and hydrocarbons), which are incapable of forming $\mathrm{H}$-bonds with water molecules, tending to aggregate or cluster when placed in water and, in doing so, to exclude water molecules. Precise details of the origins and nature of these complex phenomena are not yet fully resolved, even although both hydrophobic interactions and the hydrophobic effect have several manifestations other than the immiscibility of nonpolar solutes in water (e.g., adhesion between hydrophobic surfaces, micelle formation, and protein folding) and have been the subject of a substantial amount of research over many decades. In the first discussion of the hydrophobic effect, Frank and Evans proposed that water molecules formed "microscopic icebergs" around a nonpolar molecule, with this rearrangement of the water molecules being driven entropically. In essence, the introduction of a nonpolar molecule into water disrupts the three-dimensional, extensively $\mathrm{H}$-bonded network of water molecules and imposes a new and more ordered structure on the water molecules [15]. As a result of this entropic allied unflavored arrangement, the water molecules reorient to minimize their contact with the nonpolar molecules and reduce the disruption of the H-bonded network. The nonpolar molecules aggregate to reduce their contact with the water molecules and minimize their disruptive effect and, in doing so, eject entropically unflavored water molecules, thereby reducing the total free energy of the system. However, more recent research suggests that hydrophobic interactions can be either entropy- or enthalpydriven. Indeed, several (equivalent) explanations have been proposed to describe the structure of water at hydrophobic surfaces, ranging from one in which a water molecule at the surface loses one of the $\mathrm{H}$-bonds that it shares with other water molecules to the one in which water molecules that surround a nonpolar surface encase the nonpolar molecules within clathrate pentagons, thereby avoiding loss of H-bonds. Such clathrate structures comprise water molecules that are more ordered than, but whose H-bonds are no stronger than, those of the bulk water. The increased order of the water that surrounds no polar solute is considered responsible for the negative entropy change ( $-\Delta S$ change) that accompanies hydrophobic hydration. The clathrate structures are labile and are envisaged as minimum enthalpy assemblies rather than rigid "cage-like" clusters which are readily disrupted by kinetic effects (e.g., temperature). The size and shape of nonpolar solutes influence the structure of the surrounding water molecules [16]. Water molecules can more easily rearrange around small nonpolar molecules without the loss of $\mathrm{H}$-bonds and may interact via multiple van der Waals interactions, the latter being likely reflected in the characteristic negative enthalpy change ( $-\Delta H$ change) that attends hydrophobic hydration. Although various models have been proposed to explain hydrophobic interactions, little is known about these highly complex and very strong attractive forces that operate between hydrophobic surfaces. Hydrophobic interactions are considered effective over the range from $8-10 \mathrm{~nm}$ to $300 \mathrm{~nm}$, although the true hydrophobic interactions may well only occur at distances [17].

2.2. Dye-Fiber Substantively. A discussion of the findings of several workers concluded that as with all dye types on all fiber types, substantively the uses of dispersed dyes towards PET fibers can be attributed to a variety of intermolecular forces and that $\mathrm{H}$-bonding predominates, although Vander Waals forces also contribute. As with other classes of dyes, relatively small differences in the structure of a dispersed dye can have marked effects on the dye-fiber substantively. Several authors have sought to correlate dye structure to dyeing behavior using the solubility parameter concept [15].

2.2.1. Disperse Dye. A disperse dye is defined as a substantially water-insoluble dye having substantively for one or more hydrophobic fibers, for example, polyester fibers [18]. Disperse dyes are water-insoluble nonionic dyes used to dye manmade fibers from the acidic bath, specially designed for dyeing of polyester and are applied along with a dispersing agent to retain dyes in fine dispersion. Due to their application, invariably at higher temperatures, a part of the dye may get sublimated necessitating their classification based on stability against sublimation. Dyes are retained by the fiber by physical forces. Fastness properties are excellent on polyester fabric [19]. The mechanism of the adsorption of dispersed dyes, which is identical for all types of fiber for hydrophobic. The dyes are finely ground in the presence of a dispersing agent, and then sold as a paste or spray-dried and sold as a powder [20]. They can be also utilized for dyeing of nylon, triacetate, polyester, and acrylic fibers. In some cases, a dyeing temperature of $130^{\circ} \mathrm{C}$ is required and a pressurized dye bath is used. The fine molecule size gives a large surface area that helps solubility to allow dye uptake by the fiber molecule. The dyeing rate can be significantly influenced by the type of dispersing agent used during the grinding process. Since Disperse dyes have low dissociation in water, they can interact with the polyester chains despite the formation of dispersed particles. The main usage is the dyeing of polyesters, and it uses minimum dyeing for cellulose acetate and polyamide fiber types. The chemical structure of disperse dyes is small, planar, nonionic, that attaches to the functional group of $-\mathrm{NO}_{2}$ and $-\mathrm{CN}$. The structure makes it easier for the dye to slide between the tightly packed polymer molecule and the polar groups can improve the water solubility, the dipolar bonding between dye and polymer, and affect the color type of the dye. Nevertheless, their small size molecules of dispersed dyes are 
quite volatile and tend to sublime out of the polymer at adequately high temperatures. The dye is generally applied under pressure at temperatures of about $130^{\circ} \mathrm{C}$. At this temperature, thermal agitation causes the polymer's molecular structure to become amorphous and less crystalline, opening gaps for the dye molecules to enter. The interactions between the disperse dye molecules and the polyester polymers are considered van der Waals and dipole forces. The instability of the dye can cause loss of the density of color and staining of other materials at maximum temperatures. This can be countered by using bigger dye molecules or it gives the dye more polar (or both). This has a drawback, however, in that this new larger, more polar molecule will need more extreme forcing conditions to dye the polymer [16].

Acute toxicity involves oral ingestion and inhalation, skin and eye irritation, and skin sensitization. The main problems of acute toxicity with textile dyes are skin irritation and skin sensitization, caused mainly by reactive dyes for cotton and viscose, and dispersed dyes for polyester [21].

2.3. Basic Principle Disperse Dye in PET Dyeing. The inclusion of a dispersing agent in the dye bath is a crucial factor in the application of dispersed dyes. A compound is added to water, its dual character results in the formation of micelles in critical, but low concentration. The hydrophobic tails of the dispersing agent molecules are inside the micelle, which, as a consequence, is able to solubilize the disperse dye molecules, so conferring a higher apparent solubility for the dye. The dye transfer to the fiber from the micelles as micelles empty their dye, they reform and dissolve more dye from the solid particles [21]. Much of the evidence that is available on the subject suggests that in dyed polyester fibers the dispersed dyes are present chiefly in the monomolecular state. At the end of the dyeing process, the dye that has been absorbed by the fiber is in a state of dynamic equilibrium with the dye that remains in the bath, and the fraction of the latter, that is in aqueous solution must be present in the same state of aggregation as the dye in the fiber (Figure 2). It is reasonable to infer that the transfer of the dye to the fiber occurs in a monomolecular aqueous solution, the concentration of which is maintained during the first phase of the dyeing process by the progressive dissolution of solid dispersed dye from the particles size in dispersion in the dye bath (Figure 1). In the presence of dispersing agents, the following equilibrium situation is set up [22]. Polyester is nonionic and is dyed with only disperse dye; anionic dyeable polyester can easily be dyed at or below boil with direct, reactive, vat dyes, etc., where the cationic dyeable types can be dyed with basic dyes [19].

2.4. Dyeing of Polyester Fibers (PET) with Dispersed Dyes. PET fibers are dyed using dispersed dyes, which provide a wide range of hues and display generally excellent build-up and excellent fastness properties of the fibers, although some dye combinations can display anomalous fastness to light and the behavior of the dyes to heat treatment is of importance. The dyes possess low, but essential, aqueous

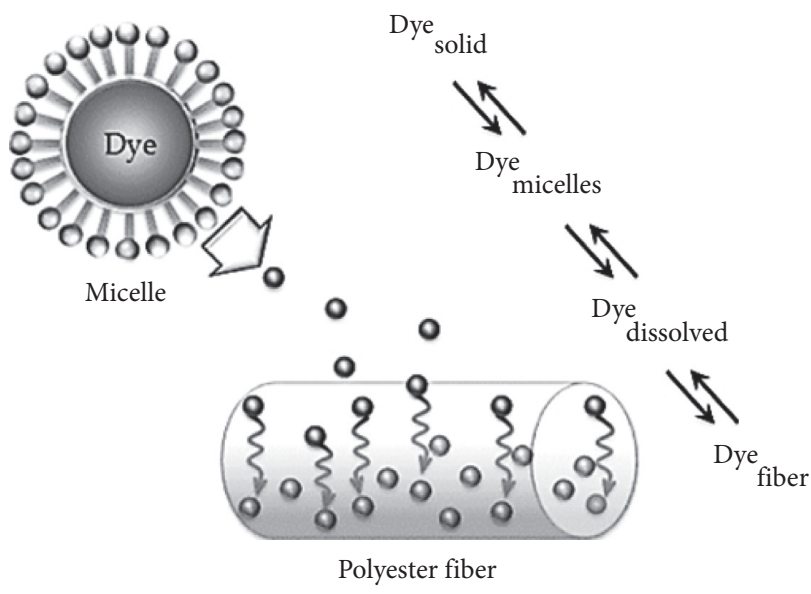

Figure 2: Disperse dyeing mechanism for polyester fibers [20].

solubility that enables them to be applied to PET (and other fibers) in the form of a fine aqueous dispersion in the presence of dispersing agents. However, owing to the low diffusion rate of dispersed dyes within PET fibers at temperatures up to $100^{\circ} \mathrm{C}$, commercially acceptable rates of dyeing are achieved either using fiber plasticizers or carriers [23], at the commercial boil $\left(100^{\circ} \mathrm{C}\right)$ in a process referred to as carrier dyeing or under pressure in the region of $130-140^{\circ} \mathrm{C}$ in a process referred to as high temperature (HT) dyeing. The nonionic, low molar mass dye molecules are volatile, a property that is utilized in their application in the vapor phase, such a process commonly being referred to as vapor phase or thermo sol dyeing, at the commercial boil $\left(100^{\circ} \mathrm{C}\right)$ in a process referred to as carrier dyeing or under pressure in the region of $130-140^{\circ} \mathrm{C}$ in a process referred to as high temperature (HT) dyeing. The nonionic, low molar mass dye molecules are volatile, a property that is utilized in their application in the vapor phase, such a process commonly being referred to as vapor phase or thermo sol dyeing.

2.4.1. Anionic and Cationic Dyes for Polyester. Since much polyester was originally used in blends with wool, it was natural that attempts should be made to modify PET to make it acid-dyeable with anionic dyes. The most popular theme was to incorporate basic additives by copolymerizing an amino hydroxyl compound or amino acid into the PET structure [24]. All such attempts failed because the copolymers were discolored yellow or brown. It was found, however, that certain polyamides, containing additional inchain tertiary amine groups, when the melt blended with PET and high-molecular-weight formed a three-phase mixture in which the polyamide was dispersed inside the PEG and this, in turn, was dispersed inside the PET. Thus, the critical components were prevented from intermixing in the melt. The mixture was melted pun successfully into fibers. Diamond-patterned fabrics were jacquard knitted with mixtures of dye variant fibers and normal PET. These could be cross-dyed to give patterned effects from a single dye bath containing both acid and disperse dyes. However, the process was deemed too complex and expensive for a 


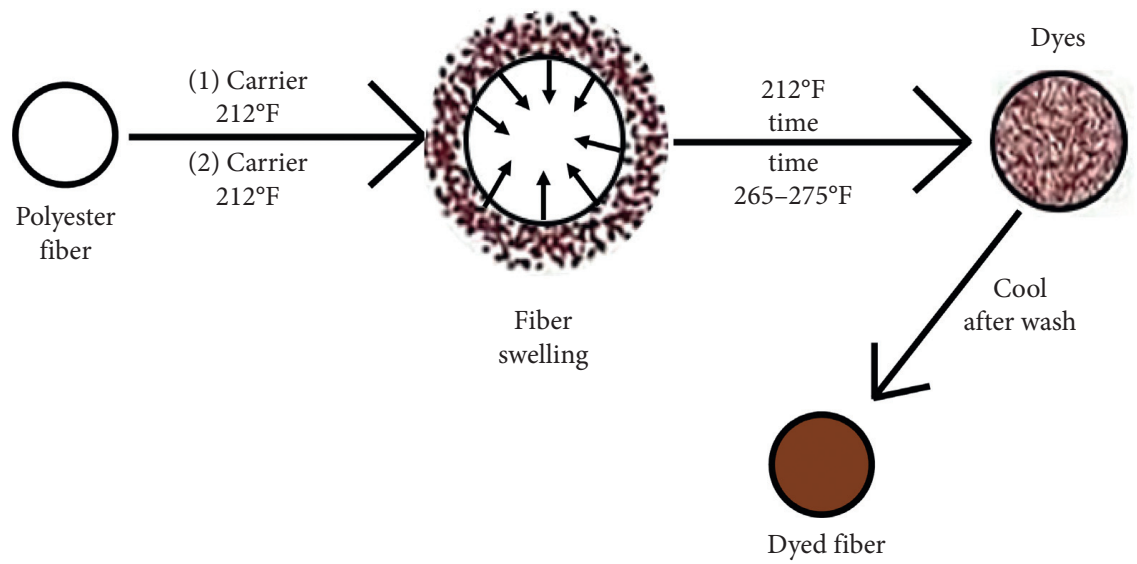

Figure 3: Representation of the mechanism of the aqueous phase transfer of dispersed dyes to hydrophobic fibers.

commercial product and the light stability of the dyes was not adequate [25]. Greater success was achieved by DuPont who copolymerized the sodium salt of 5 -sulfoisophthalic acid with PET to render the polymer dyeable to cationic (basic) dyes. Basic dyeable PET was successfully launched as Dacron 64 in the form of a low-pill staple product [14]. The presence of sulphonate groups in the polymer chain also acts as anionic dipolar cross-link and increases the melt viscosity of the polymer quite markedly. Thus, it is possible to use a melt-spin polymer with IV 0.56 under normal conditions, giving a low-pill fiber variant. The fiber also has a greater affinity for dispersed dyes due to the disruption of the PET structure. Continuing this theme, there are "deep dyes" variant PET fibers, often used in PET carpet yarns, which are copolymers of PET with chain-disrupting copolymer units like polyethylene adipate. They have less crystallinity and a lower Tg; therefore, they may be dyed at the boil without the use of pressure equipment or carrier at the cost of some loss of fiber physical properties [26].

\section{Mechanism of Dye Transfer}

The mechanism of the disperse dyeing of a hydrophobic fiber such as PET (or PA, CTA, or indeed any type of fiber) can thus be considered to comprise five sequential stages, of which the last is commonly considered as being the ratedetermining step [27]:

(1) Dissolution of dye molecules from the surface of dispersed dye particles and the establishment of a monomolecular state in the aqueous dye bath

(2) Convective diffusion of the dissolved dye molecules through the bulk dye bath to the fiber surface

(3) Diffusion of the dye molecules through the diffusion boundary layer present at the fiber surface

(4) Adsorption of the dye molecules onto the surface of the substrate

(5) Diffusion of the dye molecules within the fiber interior

The main mechanism of dyeing is to swell the fiber so that the dye molecule gets enough space to stay inside the fiber. In hydrophilic fibers such as cotton, this swelling is done by wetting. However, when we dye polyester fiber, due to the compact structure, it is not able to swell on wetting [28]. Therefore, it is a hydrophobic fiber. Its Moisture Regain percentage is $0.04 \%$, so it is highly hydrophobic. Thus, it needs a special arrangement. Swelling is done by applying chemicals or heat. The chemical swells the fiber is called carrier. Heating of the dye liquor swells the fiber to open up and assists the dye to penetrate the fiber polymer system. Thus, the dye molecule takes its place in the amorphous regions of the fiber (Figure 3 ). Once taking place within the fiber polymer system, the dye molecules are held by hydrogen bonds and van Der Waals' force. When the system is taken off, the molecular areas shrunk and dye molecules are entrapped inside the polymer [29]. Disperse dyes has a small general structure, planar and nonionic, with attached polar functional groups like $-\mathrm{NO}_{2}$ and $-\mathrm{CN}$. Dye particles size ranging from 0.5 to 2.0 micron, generally contain $-\mathrm{NH}_{2}$, substituted $-\mathrm{NH}_{2}$, or $-\mathrm{OH}$ groups in structure and are attached with fiber through $\mathrm{H}$-bond and van der Waals force [30].

\section{Kinetics of Dyeing}

The rate of uptake of dispersed dyes into PET fibers has received considerable attention and detailed accounts are available [31]. In essence, the diffusion behavior of dispersed dyes within PET and other types of fiber is almost ideal and analysis of dyeing rates has been undertaken using equations that relate to both finite and infinite dye baths. Such virtual ideal diffusion behavior has been attributed to a low degree of dye-fiber interaction that enables the ready movement of dye molecules through the substrate [32].

Confocal laser scanning microscopy has been utilized to study the diffusion of commercial AQ and benzo difuranone dispersed dyes within PET fibers. The results obtained for C.I. Disperse Red 60 and the benzodifuranone dye (Figure 4) clearly illustrate the rate at which such typical disperse dyes diffuse within the substrate as a function of both temperature and duration of dyeing. It is clear that in the early stages of dyeing, dye uptake is confined to the periphery of the fiber and that little, if any dyeing occurs $<100^{\circ} \mathrm{C}$ and, also that 
<smiles>Nc1c(Oc2ccccc2)cc(O)c2c1C(=O)c1ccccc1C2=O</smiles>

(a)

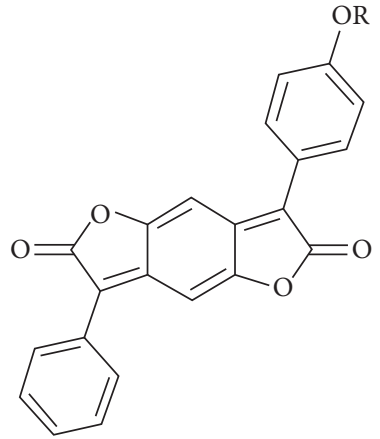

(b)

Figure 4: (a) Disperse red 60 and (b) the benzodifuranone dye I.

between 100 and $120^{\circ} \mathrm{C}$, dye uptakes are confined to the outside regions of the fiber. Furthermore, the AQ dye displays a greater rate of dyeing than its benzodifuranone counterpart, the latter requiring much longer at $130^{\circ} \mathrm{C}$ to fully penetrate the fiber [32].

\section{Conclusion}

Intermolecular force is an attraction between dye molecules. The reaction between disperse dyes and polyester molecules must take into account the various types of forces exerted. In the absence of any physical interaction, dyes are only mechanically retained. This may be due to insolubilization of the dye inside the fiber or may be due to self-association into possibly quite large molecular aggregates following their entry into the fiber. PET has no functional groups to give affinity for usual dyestuffs. Natural fibers like wool, cotton, silk, and then later manmade ones like rayon and nylon were well known and had good dye affinity because of the fibers had pendant or terminal functional chemical groups such as $-\mathrm{NH}_{2},-\mathrm{COOH}$, and $-\mathrm{OH}$. These dyes were developed to interact with such groups. This dye had little or no affinity for PET. Substantively of dispersed dyes towards PET fibers can be attributed to a variety of intermolecular forces and that $\mathrm{H}$-bonding predominates, although van der Waals forces also contribute. As with other classes of dyes, relatively small differences in the structure of a dispersed dye can have marked effects on the dye-fiber substantively. Several authors have sought to correlate dye structure to dyeing behavior using the solubility parameter concept.

\section{Data Availability}

We have annotated the entire data building process and empirical techniques presented in the paper, and other related data are assessed or reviewed from related research articles. The data that support the findings of this study are included within the manuscript references and are available from the corresponding author and online, upon reasonable requests (e-mail: amareworku2005@ddu.edu.et, phone number: +251911006574). All data used for this review can be shared online for publicity and accessibility if you recommend the website.

\section{Conflicts of Interest}

Asnake Ketema is currently working as Lecturer in Textile Chemistry at Dire Dawa Institute of Technology, Dire Dawa University, Dire Dawa, Ethiopia. Amare Worku is currently working as Lecturer in Textile Chemistry and school dean of the School of Textile, Apparel and Fashion Design at Dire Dawa Institute of Technology, Dire Dawa University, Dire Dawa, Ethiopia. All the authors declare that there are no conflicts of interest regarding the publication of this review paper.

\section{References}

[1] E. Reigel and J. Kent, Riegel's, Hendbook of Industrial Chemsitry, Vol. 1, Springer, New York, NY, USA, 2003.

[2] B. L. Deopura, R. Alagirusamy, M. Joshi, and Gupta, Polyesters and Polyamides, Vol. 71, Woodhead Publishing in Textiles, Cambridge, UK, 2008.

[3] H. Yamashita and Nakano, Polyester Properties Preparaton and Applications, NovaScience Publishers, New York, NY, USA, 2008.

[4] C.-C. Lai and K.-M. Chen, "Dyeing properties of modified gemini surfactants on a disperse dye-polyester system," Textile Research Journal, vol. 78, no. 5, pp. 382-389, 2008.

[5] M. Muneer, M. Zuber, and N. Akhtar, "Eco-friendly disperse dyeing of ultraviolet-treated polyester fabric using disperse yellow 211," Journal of Enviromental Studies, vol. 27, no. 5, pp. 1935-1939, 2018.

[6] M. Elapasery, R. A. Abdelghaffar, M. M. Kamel, M. M. Kamel, B. M. Youssef, and K. M. Haggag, "Microwave, ultrasound assisted dyeing," in Proceedings of the 8th International Conference of The Textile Research Division (ICTRD 2017), vol. 60, pp. 143-151, Cairo, Egypt, September 2017.

[7] I. A. Bhatti, S. Adeel, S. Parveen, and M. Zuber, "Dyeing of UV irradiated cotton and polyester fabrics with multifunctional reactive and disperse dyes," Journal of Saudi Chemical Society, vol. 20, no. 2, pp. 178-184, 2016.

[8] S. Adeel, M. Zuber, and M. Saeed, "Sustainable dyeing of microwave treated polyester fabric using disperse yellow 211 dye," Journal of the Mexican Chemical Society, vol. 62, no. 1, 2018.

[9] S. Dhouib, A. Lallam, and F. Sakli, "Study of dyeing behavior of polyester fibers with disperse dyes," Textile Research Journal, vol. 76, no. 4, pp. 271-280, 2006. 
[10] J. Chakraborty, Fundamentals and Practices in Colouration of Textiles, Woodhead Publishing, New Delhi, India, 2015.

[11] H. Najafi, R. Assefipour, M. Hajilari, and H. R. Movahed, "One bath method dyeing of polyester/cotton blend fabric with sulphatoethylsulphonyl disperse/reactive dyes treatment by chitin biopolymer," African Journal of Biotechnology, vol. 8, no. 6, pp. 1127-1135, 2009.

[12] G. Oyeleke, A. Isola, W. Sulaiman, and A. Adebisi, "Dye uptake of polyethylene terephthalate fiber in non-aqueous solvent," Biopolymers Research, vol. 1, no. 102, p. 2, 2017.

[13] S. Burkinshaw, Chemical Principles of Synthetic Fibre Dyeing, Springer Science \& Business Media, Berlin, Germany, 1995.

[14] J. E. McIntyre, Synthetic Fibres: Nylon, Polyester, Acrylic, Polyolefin, Woodhead, Cambridge, UK, 2005.

[15] M. Lewin and E. M. Pearce, Handbook of Fiber Chemistry, CRC Press, Boca Raton, FL, USA, 1998.

[16] W. McDowell and R. Weingarten, "New experimental evidence about the dyeing of polyester materials with disperse dyes," Textile Research Journal, vol. 85, no. 12, pp. 589-597, 1969.

[17] H. M. Mashaly, A. Rehab, M. K. Mona, and B. M. Youssef, "Dyeing of polyester fabric using nano disperse dyes and improving their light fastness using $\mathrm{ZnO}$ nano powder," Indian Journal of Science and Technology, vol. 7, no. 4, pp. 960-967, 2014.

[18] T.-S. Choi, Y. Shimizu, H. Shirai, and K. Hamada, "Disperse dyeing of polyester fiber using Gemini surfactants containing ammonium cations as auxiliaries," Dyes and Pigments, vol. 50, no. 1, pp. 55-65, 2001.

[19] D. J. Brunelle, "Synthesis and polymerization of cyclic polyester oligomers," Modern Polyesters: Chemistry and Technology of Polyesters and Copolyesters, Wiley Online Library, Hoboken, NJ, USA, 2004.

[20] G. Vassilatos, B. H. Knox, and H. R. E. Frankfort, "Dynamics, structure development, and fiber properties in high-speed spinning of polyethylene terephthalate," Society of Dyer and Colorist, Coloration Technology, 1985, https://scholar.google. com/scholar?hl=en\&as_sdt=0\%2C5\&q=Dynamics $\% 2 \mathrm{C}+$ structure+ development $\% 2 \mathrm{C}+$ and + fiber+properties + in + high-speed + spinning+of+polyethylene+terephthalate\&btnG=

[21] R. M. Christie, Environmental Aspects of Textile Dyeing, Elsevier, Amsterdam, Netherlands, 2007.

[22] M. Shahin, R. Ahmed, and M. M. Marie, "Optimizing the dyeing process of alkali-treated polyester fabric with dolu natural dye," Journal of Engineering Research and Applications, vol. 4, no. 6, pp. 35-40, 2014.

[23] I. M. Ward, "The molecular structure and mechanical properties of polyethylene terephthalate fibers," Textile Research Journal, vol. 7, no. 31, pp. 650-664, 1961.

[24] B. H. Knox, H.-D. Weigmann, and M. G. Scott, "Interactions of nonaqueous solvents with textile fibers: part V: application of the solubility parameter concept to polyester fiber-solvent interactions," Textile Research Journal, vol. 3, no. 45, pp. 203-217, 1975.

[25] A. D. Broadbent, Basic Principles of Textile Oloration, Society of Dyers and Colourists, West Yorkshire, UK, 2001.

[26] K. H. Illers and H. Breuer, "Molecular motions in polyethylene terephthalate," Journal of Colloid Science, vol. 18, no. 1, pp. 1-31, 1963.

[27] M. El-Apasery, "Synthesis of some azo disperse dyes by the use of focused microwave heating," Journal of Applied Chemistry, vol. 50, no. 3-4, pp. 75-81, 2006.

[28] J. G. Blacker and D. Patterson, "Molecular mechanisms of disperse dyeing of polyester and nylon fibres," Journal of the
Society of Dyers and Colourists, vol. 12, no. 85, pp. 598-605, 1969.

[29] M. Z. Uddin, M. Watanabe, H. Shirai, and T. Hirai, "Dyeing conventional and microfiber polyester with disperse dyes," Textile Research Journal, vol. 72, no. 1, pp. 77-82, 2002.

[30] H. Braun, "Particle size and solubility of disperse dyes," Textile Research Journal, vol. 13, no. 1, pp. 62-72, 1983.

[31] D.-f. Yang, X.-j. Kong, D. Gao, H.-s. Cui, T.-t. Huang, and J.-x. Lin, "Dyeing of cotton fabric with reactive disperse dye contain acyl fluoride group in supercritical carbon dioxide," Dyes and Pigments, vol. 139, pp. 566-574, 2017.

[32] Z. Ren, C. Qin, R.-C. Tang, and G. Chen, "Study on the dyeing properties of hemicyanine dyes. II. Cationic dyeable polyester," Coloration Technology, vol. 128, no. 2, pp. 147-152, 2012. 